\title{
A New Extragradient-Type Algorithm for the Split Feasibility Problem
}

\author{
Yazheng Dang, Yan Gao, and Bo Wang \\ School of Management, University of Shanghai for Science and Technology, Shanghai 200093, China \\ Correspondence should be addressed to Yazheng Dang; jgdyz@163.com
}

Received 18 October 2015; Revised 30 December 2015; Accepted 24 January 2016

Academic Editor: Reza Jazar

Copyright (C) 2016 Yazheng Dang et al. This is an open access article distributed under the Creative Commons Attribution License, which permits unrestricted use, distribution, and reproduction in any medium, provided the original work is properly cited.

We consider the split feasibility problem (SFP) in Hilbert spaces, inspired by extragradient method presented by Ceng, Ansari for split feasibility problem, subgradient extragradient method proposed by Censor, and variant extragradient-type method presented by Yao for variational inequalities; we suggest an extragradient-type algorithm for the SFP. We prove the strong convergence under some suitable conditions in infinite-dimensional Hilbert spaces.

\section{Introduction}

The convex feasibility problem (CFP) is to find a common point in the intersection of finitely many convex sets. A popular approach to the CFP is a projection algorithm which employs orthogonal projections onto a set; see [1]. When there are only two sets and constraints are imposed on the solutions in the domain of a linear operator as well as in this operator's ranges, the problem is said to be a split feasibility problem (SFP) [2]. In other words, the SFP is to find a point $x$ such that

$$
\begin{array}{r}
x \in C, \\
A x \in Q,
\end{array}
$$

where $C$ and $Q$ are nonempty closed convex subsets of real Hilbert spaces $H^{1}$ and $H^{2}$, respectively, and $A: H^{1} \rightarrow H^{2}$ is a bounded linear operator. The SFP serves as a model for many inverse problems where constraints are imposed on the solutions in the domain of a linear operator as well as in this operator's range. There are a number of significant applications of the SFP in intensity-modulated radiation therapy, signal processing, image reconstruction, and others [2-4].

Various algorithms have been invented to solve the SFP; see [5-14] and references therein. In particular, Byrne [5] introduced a so-called CQ algorithm, taking an initial point $x^{0}$ arbitrarily and defining the iterative step as

$$
x^{k+1}=P_{C}\left[\left(I-\gamma A^{T}\left(I-P_{Q}\right) A\right)\left(x^{k}\right)\right],
$$

where $0<\gamma<2 / \rho\left(A^{T} A\right), \rho\left(A^{T} A\right)$ is the spectral radius of $A^{T} A$, and $P_{S}$ denotes the projection onto set $S$; that is, $P_{S}(x)=$ $\arg \min _{y \in S}\|x-y\|$.

Extragradient algorithm was first introduced by Korpelevich [15] for computing a solution of a variational inequality and shows the quick convergence. Subsequently, Nadezhkina and Takahashi in [16] applied the method for finding a common element of the set of fixed points of a nonexpansive mapping and the set of solutions of variational inequality. Ceng et al. in [17] proposed an extragradient method, and Yao et al. in [18] presented subgradient extragradient method to solve the split feasibility problem, but all these algorithms have only weak convergence in the infinite-dimensional Hilbert spaces. Hence, to obtain strong convergence, Censor et al. in [19] presented a variant extragradient-type method and Censor et al. in [20] proposed subgradient extragradient method which possesses strong convergence for solving the variational inequality. Motivated by the works given above, in this paper, we construct an extragradient-type method to solve the split feasibility problem. Strong convergence of the algorithm is proved under some suitable conditions in the infinite-dimensional Hilbert spaces. 
The paper is organized as follows. Section 2 reviews some preliminaries. Section 3 gives a variant extragradient-type algorithm and shows its convergence. Section 4 gives some conclusions.

\section{Preliminaries}

Throughout the paper, $I$ denotes the identity operator, and $\operatorname{Fix}(T)$ denotes the fixed points of an operator $T$; that is, $\operatorname{Fix}(T):=\{x \mid x=T(x)\}$.

In this section, we review some concepts and basic results that will be used later.

Definition 1. Let $T$ be a mapping from a set $C \subset H^{1}$ into $H^{1}$. Then consider the following:

(a) $T$ is said to be nonexpansive, if

$$
\|T(x)-T(y)\| \leq\|x-y\|, \quad \forall x, y \in C ;
$$

(b) $T$ is said to be firmly nonexpansive, if

$$
\begin{aligned}
& \|T(x)-T(y)\|^{2} \leq\langle x-y, T(x)-T(y)\rangle, \\
& \forall x, y \in C ;
\end{aligned}
$$

(c) $T$ is said to be contractive on $X$, if there exists $0<\alpha<$ 1 such that

$$
\|T(x)-T(y)\| \leq \alpha\|x-y\|, \quad \forall x, y \in C ;
$$

(d) $T$ is said to be monotone, if

$$
\langle T(x)-T(y), x-y\rangle \geq 0, \quad \forall x, y \in C ;
$$

(e) $T$ is said to be $\beta$-inverse strongly monotone $(\beta$-ism), if there exists a real number $\beta>0$ such that

$$
\begin{aligned}
\langle T(x)-T(y), x-y\rangle \geq \beta\|T(x)-T(y)\|^{2} & \\
& \forall x, y \in C .
\end{aligned}
$$

The lemma below is the basic properties of the projection operator $P_{C}[21]$.

Lemma 2. Let $C \subset H^{1}$ be nonempty, closed, and convex. Then, for all $x, y \in H^{1}$ and $c \in C$,

(1) $\left\langle x-P_{C}(x), c-P_{C}(x)\right\rangle \leq 0$;

(2) $\left\|P_{C}(x)-P_{C}(y)\right\|^{2} \leq\left\langle P_{C}(x)-P_{C}(y), x-y\right\rangle$;

(3) $\left\|P_{C}(x)-c\right\|^{2} \leq\|x-c\|^{2}-\left\|P_{C}(x)-x\right\|^{2}$.

From (2) of Lemma 2, we know that the projection operator $P_{C}$ is monotone and nonexpansive, and $2 P_{C}-I$ is nonexpansive.

The lemmas below are necessary for the convergence analysis in the next section.
Lemma 3 (see [22]). Let $C$ be a nonempty closed convex subset of a real Hilbert space $H$ and let $T: C \rightarrow H$ be a $\alpha$-inverse strongly monotone and let $r>0$ be a constant. Then, one has

$$
\begin{array}{ll}
\|(I-r T)(x)-(I-r T)(y)\|^{2} & \\
\quad \leq\|x-y\|^{2}+r(r-2 \alpha)\|T(x)-T(y)\|^{2} & \\
& \forall x, y \in C .
\end{array}
$$

In particular, if $0<r<2 \alpha$, then $I-r T$ is nonexpansive.

Lemma 4 (see [9]). Let $\left\{x^{k}\right\}$ and $\left\{y^{k}\right\}$ be bounded sequences in a Banach space $E$ and let $\left\{\beta_{k}\right\}$ be a sequence in $[0,1]$ which satisfies the following condition: $0<\lim _{\inf _{k \rightarrow \infty}} \beta_{k} \leq$ $\lim \sup _{k \rightarrow \infty} \beta_{k}<1$. Suppose that $x^{k+1}=\left(1-\beta_{k}\right) y^{k}+\beta_{k} x^{k}$ for all $k \geq 0$ and $\lim \sup _{k \rightarrow \infty}\left(\left\|y^{k+1}-y^{k}\right\|-\left\|x^{k+1}-x^{k}\right\|\right) \leq 0$; then $\lim _{k \rightarrow \infty}\left\|y^{k}-x^{k}\right\|=0$.

Lemma 5 (see [23, Lemma 2.1]). Let $\left\{a_{k}\right\}$ be a sequence of nonnegative real numbers satisfying the condition

$$
a_{k+1} \leq\left(1-m_{k}\right) a_{k}+m_{k} \delta_{k}, \quad \forall k \geq 0,
$$

where $\left\{m_{k}\right\},\left\{\delta_{k}\right\}$ are sequences of real numbers such that

(i) $\left\{m_{k}\right\} \subset[0,1]$ and $\sum_{k=0}^{\infty} m_{k}=\infty$, or, equivalently,

$$
\prod_{k=0}^{\infty}\left(1-m_{k}\right):=\lim _{k \rightarrow \infty} \prod_{j=0}^{k}\left(1-m_{i}\right)=0,
$$

(ii) $\lim \sup _{k \rightarrow \infty} \delta_{k} \leq 0$, or

(ii) ${ }^{\prime} \sum_{k=0}^{\infty} \delta_{k} m_{k}$ is convergent.

Then, $\lim _{k \rightarrow \infty} a_{k}=0$.

\section{Algorithm and Its Convergence Analysis}

In this section, we present the formal statement of our proposal for the algorithm. Denote the solution set of the SFP by

$$
\Gamma=\{x \in C: A x \in Q\}
$$

3.1. Variant Extragradient-Type Method. Now, we give the extragradient-type algorithm.

Algorithm 6. Let $C$ be a nonempty, closed, and convex subset of a real Hilbert space $H$. Consider the sequences $\left\{\alpha_{k}\right\} \subset$ $[0,1],\left\{\lambda_{k}\right\} \subset\left[0,2 / \rho\left(A^{T} A\right)\right],\left\{\mu_{k}\right\} \subset\left[0,2 / \rho\left(A^{T} A\right)\right]$, and $\left\{\gamma_{k}\right\} \subset[0,1]$. For an arbitrary initial point $x^{0}, x^{k}$ is the current point. Define a mapping $F: H^{1} \rightarrow H^{1}$ as

$$
F(x)=A^{T}\left(I-P_{\mathrm{Q}}\right) A x .
$$

For $k=0,1,2, \ldots$, compute

$$
\begin{aligned}
y^{k} & =P_{C}\left[\left(1-\alpha_{k}\right) x^{k}-\lambda_{k} F\left(x^{k}\right)\right], \\
x^{k+1} & =P_{C}\left[x^{k}-\mu_{k} F\left(y^{k}\right)+\gamma_{k}\left(y^{k}-x^{k}\right)\right], \quad k \geq 0 .
\end{aligned}
$$


3.2. Convergence Analysis. In the section, we consider the convergence analysis of Algorithm 6.

Theorem 7. Suppose that $\Gamma \neq \emptyset$. Assume that the algorithm parameters $\left\{\alpha_{k}\right\},\left\{\lambda_{k}\right\},\left\{\mu_{k}\right\}$, and $\left\{\gamma_{k}\right\}$ satisfy the following conditions:

(C1) $\lim _{k \rightarrow \infty} \alpha_{k}=0$ and $\sum_{k=1}^{\infty} \alpha_{k}=\infty$;

(C2) $\lambda_{k} \in[a, b] \subset\left(0,2 / \rho\left(A^{T} A\right)\right)$ and $\lim _{k \rightarrow \infty}\left(\lambda_{k+1}-\lambda_{k}\right)=$ 0 ;

(C3) $\gamma_{k} \in(0,1), \mu_{k} \leq\left(2 / \rho\left(A^{T} A\right)\right) \gamma_{k}$, and $\lim _{k \rightarrow \infty}\left(\gamma_{k+1}-\right.$ $\left.\gamma_{k}\right)=\lim _{k \rightarrow \infty}\left(\mu_{k+1}-\mu_{k}\right)=0$;

(C4) $\sum_{k=1}^{+\infty}\left(\gamma_{k} / \lambda_{k}\right)<\infty$.

Then, the sequences $\left\{x^{k}\right\}$ generated by Algorithm 6 converge strongly to a point in $\Gamma$.

Proof. Picking $z \in \Gamma$, we divide the proof into several steps.

(1) First, we prove that $\left\{x^{k}\right\},\left\{y^{k}\right\},\left\{F\left(x^{k}\right)\right\}$, and $\left\{F\left(y^{k}\right)\right\}$ are all bounded.

By conditions (C1) and (C2), since $\alpha_{k} \rightarrow 0$ and $\lambda_{k} \epsilon$ $\left(0,2 / \rho\left(A^{T} A\right)\right)$, we have $\alpha_{k}<1-\lambda_{k} \rho\left(A^{T} A\right) / 2$, as $k \rightarrow \infty$. Hence, we may assume that, for all $k, \alpha_{k}<1-\lambda_{k} \rho\left(A^{T} A\right) / 2$; then, $\lambda_{k} /\left(1-\alpha_{k}\right) \in\left(0,2 / \rho\left(A^{T} A\right)\right)$. By the property of the projection, we know $z=P_{C}[z-t F(z)]$ for any $t>0$. Hence,

$$
\begin{aligned}
z & =P_{C}\left[z-\frac{\lambda_{k}}{1-\alpha_{k}} F(z)\right]=P_{C}\left[z-\lambda_{k} F(z)\right] \\
& =P_{C}\left[\alpha_{k} z+\left(1-\alpha_{k}\right)\left(z-\frac{\lambda_{k}}{1-\alpha_{k}} F(z)\right)\right],
\end{aligned}
$$

$\forall k \geq 0$;

that is,

$$
z=P_{C}\left[\alpha_{k} z+\left(1-\alpha_{k}\right)\left(z-\frac{\lambda_{k}}{1-\alpha_{k}} F(z)\right)\right]
$$

$\forall k \geq 0$

Thus, by (12b) and (14), we have

$$
\begin{aligned}
& \left\|y^{k}-z\right\|=\left\|P_{C}\left[\left(1-\alpha_{k}\right) x^{k}-\lambda_{k} F\left(x^{k}\right)\right]-z\right\| \\
& =\| P_{C}\left[\left(1-\alpha_{k}\right)\left(x^{k}-\frac{\lambda_{k}}{1-\alpha_{k}} F\left(x^{k}\right)\right)\right] \\
& -P_{C}\left[\alpha_{k} z+\left(1-\alpha_{k}\right)\left(z-\frac{\lambda_{k}}{1-\alpha_{k}} F(z)\right)\right] \| \\
& \leq \|-\alpha_{k} z+\left(1-\alpha_{k}\right) \\
& \cdot\left[x^{k}-\frac{\lambda_{k}}{1-\alpha_{k}} F\left(x^{k}\right)-z+\frac{\lambda_{k}}{1-\alpha_{k}} F(z)\right] \| .
\end{aligned}
$$

Since $\lambda_{k} /\left(1-\alpha_{k}\right) \in\left(0,2 / \rho\left(A^{T} A\right)\right)$, from Lemma 3, we know that $I-\left(\lambda_{k} /\left(1-\alpha_{k}\right)\right) F$ is nonexpansive. From the above inequality, we have

$$
\left\|y^{k}-z\right\| \leq \alpha_{k}\|-z\|+\left(1-\alpha_{k}\right)\left\|x^{k}-z\right\| .
$$

By (C3), we obtain $\mu_{k} / \gamma_{k} \leq 2 / \rho\left(A^{T} A\right)$. So, $I-\left(\lambda_{k} /\left(1-\alpha_{k}\right)\right) F$ is nonexpansive. Therefore,

$$
\begin{aligned}
& \left\|x^{k+1}-z\right\|=\left\|P_{C}\left[x^{k}-\mu_{k} F\left(y^{k}\right)+\gamma_{k}\left(y^{k}-x^{k}\right)\right]-z\right\| \\
& =\| P_{C}\left[\left(1-\gamma_{k}\right) x^{k}+\gamma_{k}\left(y^{k}-\frac{\mu_{k}}{\gamma_{k}} F\left(y^{k}\right)\right)\right] \\
& -P_{C}\left[\left(1-\gamma_{k}\right) z+\gamma_{k}\left(z-\frac{\mu_{k}}{\gamma_{k}} F(A z)\right)\right] \| \leq(1 \\
& \left.-\gamma_{k}\right)\left\|x^{k}-z\right\|+\gamma_{k} \| y^{k}-\frac{\mu_{k}}{\gamma_{k}} F\left(y^{k}\right) \\
& -\left(z-\frac{\mu_{k}}{\gamma_{k}} F(z)\right)\left\|\leq\left(1-\gamma_{k}\right)\right\| x^{k}-z\left\|+\gamma_{k}\right\| y^{k} \\
& -z\left\|\leq\left(1-\gamma_{k}\right)\right\| x^{k}-z\left\|+\gamma_{k} \alpha_{k}\right\|-z \|+\gamma_{k}\left(1-\alpha_{k}\right) \\
& \cdot\left\|x^{k}-z\right\|=\left(1-\gamma_{k} \alpha_{k}\right)\left\|x^{k}-z\right\|+\gamma_{k} \alpha_{k}\|-z\| \\
& \leq \max \left\{\left\|x^{k}-z\right\|,\|-z\|\right\} .
\end{aligned}
$$

By induction, we get

$$
\left\|x^{k+1}-z\right\| \leq \max \left\{\left\|x^{0}-z\right\|,\|-z\|\right\}
$$

Then $\left\{x^{k}\right\}$ is bounded, and so are $\left\{y^{k}\right\},\left\{F\left(x^{k}\right)\right\}$, and $\left\{F\left(y^{k}\right)\right\}$.

(2) We claim that $\lim _{k \rightarrow \infty}\left\|x^{k+1}-x^{k}\right\|=0$ and $\lim _{k \rightarrow \infty}\left\|x^{k}-y^{k}\right\|=0$.

Let $S=2 P_{C}-I$. From the property of the projection, we know that $S$ is nonexpansive. Therefore, we can rewrite $x^{k+1}$ in $(12 \mathrm{c})$ as

$$
\begin{aligned}
x^{k+1}= & \frac{I+S}{2}\left[\left(1-\gamma_{k}\right) x^{k}+\gamma_{k}\left(y_{k}-\frac{\mu_{k}}{\gamma_{k}} F\left(y^{k}\right)\right)\right] \\
= & \frac{1-\gamma_{k}}{2} x^{k}+\frac{\gamma^{k}}{2}\left(y_{k}-\frac{\mu_{k}}{\gamma_{k}} F\left(y^{k}\right)\right) \\
& +\frac{1}{2} S\left[\left(1-\gamma_{k}\right) x^{k}+\gamma_{k}\left(y_{k}-\frac{\mu_{k}}{\gamma_{k}} F\left(y^{k}\right)\right)\right] ;
\end{aligned}
$$

then

$$
x^{k+1}=\frac{1-\gamma_{k}}{2} x^{k}+\frac{1+\gamma_{k}}{2} z^{k}
$$

where 


$$
\begin{aligned}
z^{k} & =\frac{\left(\gamma^{k} / 2\right)\left(y_{k}-\left(\mu_{k} / \gamma_{k}\right) F\left(y^{k}\right)\right)+(1 / 2) S\left[\left(1-\gamma_{k}\right) x^{k}+\gamma_{k}\left(y_{k}-\left(\mu_{k} / \gamma_{k}\right) F\left(y^{k}\right)\right)\right]}{\left(1+\gamma_{k}\right) / 2} \\
& =\frac{\gamma^{k}\left(y_{k}-\left(\mu_{k} / \gamma_{k}\right) F\left(y^{k}\right)\right)+S\left[\left(1-\gamma_{k}\right) x^{k}+\gamma_{k}\left(y_{k}-\left(\mu_{k} / \gamma_{k}\right) F\left(y^{k}\right)\right)\right]}{1+\gamma_{k}} .
\end{aligned}
$$

It follows that

$$
\begin{aligned}
z^{k+1}-z^{k}= & \frac{\gamma^{k+1}\left(y_{k+1}-\left(\mu_{k+1} / \gamma_{k+1}\right) F\left(y^{k+1}\right)\right)+S\left[\left(1-\gamma_{k+1}\right) x^{k+1}+\gamma_{k+1}\left(y_{k+1}-\left(\mu_{k+1} / \gamma_{k+1}\right) F\left(y^{k+1}\right)\right)\right]}{1+\gamma_{k+1}} \\
& -\frac{\gamma^{k}\left(y_{k}-\left(\mu_{k} / \gamma_{k}\right) A^{T}\left(I-P_{\mathrm{Q}}\right) A y^{k}\right)+S\left[\left(1-\gamma_{k}\right) x^{k}+\gamma_{k}\left(y_{k}-\left(\mu_{k} / \gamma_{k}\right) F\left(y^{k}\right)\right)\right]}{1+\gamma_{k}} .
\end{aligned}
$$

Hence,

$$
\begin{aligned}
& \left\|z^{k+1}-z^{k}\right\| \leq \frac{\gamma_{k+1}}{1+\gamma_{k+1}} \|\left(y_{k+1}-\frac{\mu_{k+1}}{\gamma_{k+1}} F\left(y^{k+1}\right)\right) \\
& -\left(y_{k}-\frac{\mu_{k}}{\gamma_{k}} F\left(y^{k}\right)\right)\left\|+\left|\frac{\gamma_{k+1}}{1+\gamma_{k+1}}-\frac{\gamma_{k}}{1+\gamma_{k}}\right|\right\| y_{k} \\
& -\frac{\mu_{k}}{\gamma_{k}} F\left(y^{k}\right) \|+\frac{1}{1+\gamma_{k+1}} S\left[\left(1-\gamma_{k+1}\right) x^{k+1}\right. \\
& \left.+\gamma_{k+1}\left(y^{k+1}-\frac{\mu_{k+1}}{\gamma_{k+1}} F\left(y^{k+1}\right)\right)\right]-S\left[\left(1-\gamma_{k}\right) x^{k}\right. \\
& \left.+\gamma_{k}\left(y^{k}-\frac{\mu_{k}}{\gamma_{k}} F\left(y^{k}\right)\right)\right]+\left|\frac{1}{1+\gamma_{k+1}}-\frac{1}{1+\gamma_{k}}\right| \\
& \text {. }\left\|S\left[\left(1-\gamma_{k}\right) x^{k}+\gamma_{k}\left(y^{k}-\frac{\mu_{k}}{\gamma_{k}} F\left(y^{k}\right)\right)\right]\right\| \\
& \leq \frac{\gamma_{k+1}}{1+\gamma_{k+1}}\left\|\left(I-\frac{\mu_{k+1}}{\gamma_{k+1}} F\right) y^{k+1}-\left(I-\frac{\mu_{k}}{\gamma_{k}} F\right) y^{k}\right\| \\
& +\frac{\gamma_{k+1}}{1+\gamma_{k+1}}\left|\frac{\mu_{k+1}}{\gamma_{k+1}}-\frac{\mu_{k}}{\gamma_{k}}\right|\left\|F\left(y^{k}\right)\right\|+\mid \frac{\gamma_{k+1}}{1+\gamma_{k+1}} \\
& -\frac{\gamma_{k+1}}{1+\gamma_{k+1}} \mid\left\|y^{k}-\frac{\mu_{k}}{\gamma_{k}} F\left(y^{k}\right)\right\| \\
& +\frac{1}{1+\gamma_{k+1}} \| S\left[\left(1-\gamma_{k+1}\right) x^{k+1}\right. \\
& \left.+\gamma_{k+1}\left(y^{k+1}-\frac{\mu_{k+1}}{\gamma_{k+1}} F\left(y^{k+1}\right)\right)\right]-S\left(1-\gamma_{k}\right) x^{k} \\
& +\gamma_{k}\left(y^{k}-\frac{\mu_{k}}{\gamma_{k}} F\left(y^{k}\right)\right) \|+\left|\frac{1}{1+\gamma_{k+1}}-\frac{1}{1+\gamma_{k}}\right| \\
& \text {. }\left\|S\left[\left(1-\gamma_{k}\right) x^{k}+\gamma_{k}\left(y^{k}-\frac{\mu_{k}}{\gamma_{k}} F\left(y^{k}\right)\right)\right]\right\| .
\end{aligned}
$$

Again, by using the nonexpansivity of $I-\left(\mu_{k} / \gamma_{k}\right) F$ and $S$, we have

$$
\begin{aligned}
& \left\|z^{k+1}-z^{k}\right\| \leq \frac{\gamma_{k+1}}{1+\gamma_{k+1}}\left\|y^{k+1}-y^{k}\right\|+\mid \frac{\gamma_{k+1}}{1+\gamma_{k+1}} \\
& -\frac{\gamma_{k}}{1+\gamma_{k}}\left|\left\|y^{k}-\frac{\mu_{k}}{\gamma_{k}} F\left(y^{k}\right)\right\|+\frac{\gamma_{k+1}}{1+\gamma_{k+1}}\right| \frac{\mu_{k+1}}{\gamma_{k+1}}-\frac{\mu_{k}}{\gamma_{k}} \mid \\
& \cdot\left\|F\left(y^{k}\right)\right\|+\frac{1}{1+\gamma^{k+1}} \|\left(1-\gamma_{k+1}\right)\left(x^{k+1}-x^{k}\right) \\
& +\gamma_{k+1}\left[\left(I-\frac{\mu_{k+1}}{\gamma_{k+1}} F\right) y^{k+1}-\left(I-\frac{\mu_{k+1}}{\gamma_{k+1}} F\right) y^{k}\right] \\
& +\left(\gamma_{k+1}-\gamma_{k}\right)\left(y^{k}-x^{k}\right)+\left(\mu_{k}-\mu_{k+1}\right) F\left(y^{k}\right) \| \\
& +\left|\frac{1}{1+\gamma_{k+1}}-\frac{1}{1+\gamma_{k}}\right| \\
& \cdot\left\|S\left[\left(1-\gamma_{k}\right) x^{k}+\gamma_{k}\left(y^{k}-\frac{\mu_{k}}{\gamma_{k}} F\left(y^{k}\right)\right)\right]\right\| \\
& \quad \leq \frac{\gamma_{k+1}}{1+\gamma_{k+1}}\left\|y^{k+1}-y^{k}\right\|+\left|\frac{\gamma_{k+1}}{1+\gamma_{k+1}}-\frac{\gamma_{k}}{1+\gamma_{k}}\right| \| y^{k} \\
& +\frac{\mu_{k}}{\gamma_{k}} F\left(y^{k}\right)\left\|+\frac{\gamma_{k+1}}{1+\gamma_{k+1}}\left|\frac{\mu_{k+1}}{\gamma_{\gamma_{k+1}}}-\frac{\mu_{k}}{\gamma_{\gamma_{k}}}\right|\right\| F\left(y^{k}\right) \| \\
& +\frac{1-\gamma_{k+1}}{1+\gamma_{k+1}}\left\|x^{k+1}-x^{k}\right\|+\frac{\gamma_{k+1}}{1+\gamma_{k+1}}\left\|y^{k+1}-y^{k}\right\| \\
& +\frac{\left|\gamma_{k+1}-\gamma_{k}\right|}{1+\gamma_{k+1}}\left(\left\|x^{k}\right\|+\left\|y^{k}\right\|\right) \\
& \left.+\gamma_{k+1}^{k}\right) \|+\left|\frac{1}{1+\gamma_{k+1}}-\frac{1}{1+\gamma_{k}}\right| \\
& \left.\left.\gamma_{k}\right) x^{k}+\gamma_{k}\left(y^{k}-\frac{\mu_{k}}{\gamma_{k}} F\left(y^{k}\right)\right)\right] \| \cdot \\
& \left.+y^{k}\right)
\end{aligned}
$$


By (12a), (12b), and (12c), we have

$$
\begin{aligned}
& \left\|y^{k+1}-y^{k}\right\|=\| P_{C}\left[x^{k+1}-\lambda_{k+1} F\left(x^{k+1}\right)-\alpha_{k+1} x^{k+1}\right] \\
& -P_{C}\left[x^{k}-\lambda_{k} F\left(x^{k}\right)-\alpha_{k} x^{k}\right] \| \\
& \leq\left\|\left[x^{k+1}-\lambda_{k+1} F\left(x^{k+1}\right)\right]-\left[x^{k}-\lambda_{k} F\left(x^{k}\right)\right]\right\| \\
& +\alpha_{k+1}\left\|-x^{k+1}\right\|+\alpha_{k}\left\|-x^{k}\right\|=\| I-\lambda_{k+1} F\left(x^{k+1}\right) \\
& -\left(I-\lambda_{k+1} F\right) x^{k}+\left(\lambda_{k}-\lambda_{k+1}\right) F\left(x^{k}\right) \| \\
& +\alpha_{k+1}\left\|-x^{k+1}\right\|+\alpha_{k}\left\|-x^{k}\right\| \leq\left\|x^{k+1}-x^{k}\right\|+\mid \lambda_{k+1} \\
& -\lambda_{k} \mid\left\|x^{k}\right\|+\alpha_{k+1}\left\|-x^{k+1}\right\|+\alpha_{k}\left\|-x^{k}\right\| .
\end{aligned}
$$

Therefore,

$$
\begin{aligned}
& \left\|z^{k+1}-z^{k}\right\| \leq\left|\frac{\gamma_{k+1}}{1+\gamma_{k+1}}-\frac{\gamma_{k}}{1+\gamma_{k}}\right|\left\|y^{k}-\frac{\mu_{k}}{\gamma_{k}} F\left(y^{k}\right)\right\| \\
& +\frac{\gamma_{k+1}}{1+\gamma_{k+1}}\left|\frac{\mu_{k+1}}{\gamma_{k+1}}-\frac{\mu_{k}}{\gamma_{k}}\right|\left\|F\left(y^{k}\right)\right\| \\
& +\frac{\left|\gamma_{k+1}-\gamma_{k}\right|}{1+\gamma_{k+1}}\left(\left\|x^{k}\right\|+\left\|y^{k}\right\|\right) \\
& +\frac{\left|\mu_{k+1}-\mu_{k}\right|}{1+\gamma_{k+1}}\left\|F\left(y^{k}\right)\right\|+\left\|x^{k+1}-x^{k}\right\| \\
& +\left|\frac{1}{1+\gamma_{k+1}}-\frac{1}{1+\gamma_{k}}\right| \| \\
& +\left\|S\left[\left(1-\gamma_{k}\right) x^{k}+\gamma_{k}\left(y^{k}-\frac{\mu_{k}}{\gamma_{k}} F\left(y^{k}\right)\right)\right]\right\| \\
& +\left|\lambda_{k+1}-\lambda_{k}\right|\left\|x^{k}\right\|+\alpha_{k+1}\left\|-x^{k+1}\right\|+\alpha_{k}\left\|-x^{k}\right\| .
\end{aligned}
$$

Since $\lim _{k \rightarrow \infty}\left(\gamma_{k+1}-\gamma_{k}\right)=0$ and $\lim _{k \rightarrow \infty}\left(\mu_{k+1}-\mu_{k}\right)=0$, we derive that

$$
\begin{aligned}
\lim _{k \rightarrow \infty}\left|\frac{\gamma_{k+1}}{1+\gamma_{k+1}}-\frac{\gamma_{k}}{1+\gamma_{k}}\right| & =0, \\
\lim _{k \rightarrow \infty}\left|\frac{\mu_{k+1}}{\gamma_{k+1}}-\frac{\mu_{k}}{\gamma_{k}}\right| & =0 .
\end{aligned}
$$

Note that $\left\{x^{k}\right\},\left\{y^{k}\right\}$, and $\left\{F\left(y^{k}\right)\right\}$ are bounded. Therefore,

$$
\limsup _{k \rightarrow \infty}\left(\left\|z^{k+1}-z^{k}\right\|-\left\|x^{k+1}-x^{k}\right\|\right) \leq 0 .
$$

From (20) and (28), by Lemma 4, we obtain

$$
\lim _{k \rightarrow \infty}\left\|z^{k}-x^{k}\right\|=0
$$

Hence,

$$
\lim _{k \rightarrow \infty}\left\|x^{k+1}-x^{k}\right\|=\lim _{k \rightarrow \infty} \frac{1+\gamma_{k}}{2}\left\|z^{k}-x^{k}\right\|=0 .
$$

From (12b), (12c), Lemma 3, and the convexity of the norm, we deduce

$$
\begin{aligned}
& \left\|x^{k+1}-z\right\|^{2} \leq\left(1-\gamma_{k}\right)\left\|x^{k}-z\right\|^{2}+\gamma_{k}\left\|y^{k}-z\right\|^{2} \\
& \leq \gamma_{k} \|-\alpha_{k} z+\left(1-\alpha_{k}\right) \\
& +\left[\left(x^{k}-\frac{\lambda_{k}}{1-\alpha_{k}} F\left(x^{k}\right)\right)-\left(z-\frac{\lambda_{k}}{1-\alpha_{k}} F(z)\right)\right] \|^{2} \\
& +\left(1-\gamma_{k}\right)\left\|x^{k}-z\right\|^{2} \leq\left(1-\alpha_{k}\right) \gamma_{k}\left[\left\|x^{k}-z\right\|^{2}\right. \\
& \left.+\frac{\lambda_{k}}{1-\alpha_{k}}\left(\frac{\lambda_{k}}{1-\alpha_{k}}-\frac{2}{\rho\left(A^{T} A\right)}\right)\left\|F\left(x^{k}\right)-F(z)\right\|^{2}\right] \\
& +\left(1-\gamma_{k}\right)\left\|x^{k}-z\right\|^{2}+\alpha_{k} \gamma_{k}\|-z\|^{2} \leq \alpha_{k} \gamma_{k}\|-z\|^{2} \\
& +\left\|x^{k}-z\right\|^{2}+\gamma_{k} a\left(\frac{b}{1-\alpha_{k}}-\frac{2}{\rho\left(A^{T} A\right)}\right) \| F\left(x^{k}\right) \\
& +F(z) \|^{2} .
\end{aligned}
$$

Therefore, we have

$$
\begin{gathered}
\gamma_{k} a\left(\frac{2}{\rho\left(A^{T} A\right)}-\frac{b}{1-\alpha_{k}}\right)\left\|F\left(x^{k}\right)-F(z)\right\|^{2} \\
\leq \alpha_{k} \gamma_{k}\|-z\|^{2}+\left\|x^{k}-z\right\|^{2}-\left\|x^{k+1}-z\right\|^{2} \\
\leq \alpha_{k} \gamma_{k}\|-z\|^{2} \\
\quad+\left(\left\|x^{k}-z\right\|+\left\|x^{k+1}-z\right\|\right)\left\|x^{k}-x^{k+1}\right\| .
\end{gathered}
$$

Since $\lim _{k \rightarrow \infty} \alpha_{k}=0, \lim _{k \rightarrow \infty}\left\|x^{k}-x^{k+1}\right\|=0$, and $\liminf _{k \rightarrow \infty} \gamma_{k} a\left(2 / \rho\left(A^{T} A\right)-b /\left(1-\alpha_{k}\right)\right)>0$, we deduce

$$
\lim _{k \rightarrow \infty}\left\|F\left(x^{k}\right)-F(z)\right\|=0
$$

By the property (ii) of the metric projection $P_{C}$, we have

$$
\begin{gathered}
\left\|y^{k}-z\right\|^{2}=\| P_{C}\left[\left(1-\alpha_{k}\right) x^{k}-\lambda_{k} F\left(x^{k}\right)\right]-P_{C}[z \\
\left.-\lambda_{k} F(z)\right] \| \leq\left\langle\left(1-\alpha_{k}\right) x^{k}-\lambda_{k} F\left(x^{k}\right)-(z\right. \\
\left.\left.-\lambda_{k} F(z)\right), y^{k}-z\right\rangle=\frac{1}{2}\left\{\| x^{k}-\lambda_{k} F\left(x^{k}\right)\right. \\
-\left(z-\lambda_{k} F(z)\right)-\alpha_{k} x^{k}\left\|^{2}+\right\| y^{k}-z \|^{2} \\
-\|\left(1-\alpha_{k}\right) x^{k}-\lambda_{k} F\left(x^{k}\right)-z-\lambda_{k} F(z)
\end{gathered}
$$


6

Mathematical Problems in Engineering

$$
\begin{aligned}
& \left.-\left(y^{k}-z\right) \|^{2}\right\} \leq \frac{1}{2}\left\{\| x^{k}-\lambda_{k} F\left(x^{k}\right)\right. \\
& \left.-\left(z-\lambda_{k} F(z)\right) \|^{2}\right\}+2 \alpha_{k}\left\|-\alpha_{k}\right\| \| x^{k}-\lambda_{k} F\left(x^{k}\right) \\
& -\left(z-\lambda_{k} F(z)\right)-\alpha_{k} x^{k}\|+\| y^{k}-z\left\|^{2}-\right\|\left(x^{k}-y^{k}\right) \\
& -\lambda_{k}\left(F\left(x^{k}\right)-F(z)\right)-\alpha_{k} x^{k} \|^{2} \\
& \leq \frac{1}{2}\left\{\|\left(x^{k}-\lambda_{k}\left(I-P_{\mathrm{Q}}\right) A x^{k}\right)\right. \\
& -\left(z-\lambda_{k}\left(I-P_{\mathrm{Q}}\right) A z\right)\left\|^{2}+\alpha_{k} M+\right\| y^{k}-z \|^{2} \\
& \left.-\left\|\left(x^{k}-y^{k}\right)-\lambda_{k}(F(z))-\alpha_{k} x^{k}\right\|\right\} \frac{1}{2}\left\{\left\|x^{k}-z\right\|^{2}\right. \\
& +\alpha_{k} M+\left\|y^{k}-z\right\|^{2}-\left\|x^{k}-y^{k}\right\|^{2}+2 \lambda_{k}\left\langle x^{k}\right. \\
& \left.\left.-y^{k}, F\left(x^{k}\right)-F(z)\right\rangle\right\}-2 \alpha_{k}\left\langle-x^{k}, x^{k}-y^{k}\right\rangle \\
& -\left\|\lambda_{k} F\left(x^{k}\right)-\lambda_{k}(F) z+\alpha_{k} x^{k}\right\|^{2} ;
\end{aligned}
$$

hence,

$$
\begin{aligned}
& \left\|y^{k}-z\right\|^{2} \\
& \leq \frac{1}{2}\left\{\left\|x^{k}-z\right\|^{2}+\alpha_{k} M+\left\|y^{k}-z\right\|^{2}-\left\|x^{k}-y^{k}\right\|^{2}\right\} \\
& \quad+2 \lambda_{k}\left\|x^{k}-y^{k}\right\|\left\|F\left(x^{k}\right)-F(z)\right\| \\
& \quad+2 \alpha_{k}\left\|-x^{k}\right\|\left\|x^{k}-y^{k}\right\|,
\end{aligned}
$$

where $M>0$ is some constant satisfying

$$
\begin{aligned}
& \sup _{k}\left\{2\left\|-x^{k}\right\|\left\|x^{k}-\lambda_{k} F\left(x^{k}\right)-\left(z-\lambda_{k} F(z)\right)-\alpha_{k} x\right\|\right\} \\
& \leq M .
\end{aligned}
$$

It follows that

$$
\begin{aligned}
\left\|y^{k}-z\right\|^{2} \leq & \left\|x^{k}-z\right\|^{2}+\alpha_{k} M-\left\|x^{k}-y^{k}\right\|^{2} \\
& +2 \lambda_{k}\left\|x^{k}-y^{k}\right\|\left\|F\left(x^{k}\right)-F(z)\right\| \\
& +2 \alpha_{k}\left\|-x^{k}\right\|\left\|x^{k}-y^{k}\right\|
\end{aligned}
$$

therefore

$$
\begin{aligned}
\left\|x^{k+1}-z\right\|^{2} \leq & \left(1-\gamma_{k}\right)\left\|x^{k}-z\right\|^{2}+\gamma_{k}\left\|y^{k}-z\right\|^{2} \\
\leq & \left\|x^{k}-z\right\|^{2}+\alpha_{k} M-\gamma_{k}\left\|x^{k}-y^{k}\right\|^{2} \\
& +2 \lambda_{k}\left\|x^{k}-y^{k}\right\|\left\|F\left(x^{k}\right)-F(z)\right\| \\
& +2 \alpha_{k}\left\|-x^{k}\right\|\left\|x^{k}-y^{k}\right\|,
\end{aligned}
$$

which implies that

$$
\begin{aligned}
\gamma_{k}\left\|x^{k}-y^{k}\right\|^{2} \leq & \left(\left\|x^{k}-z\right\|+\left\|x^{k+1}-z\right\|\right)\left\|x^{k+1}-x^{k}\right\| \\
& +\gamma_{k}\left\|y^{k}-z\right\|^{2}+\alpha_{k} M \\
& +2 \lambda_{k}\left\|x^{k}-y^{k}\right\|\left\|F\left(x^{k}\right)-F(z)\right\| \\
& +2 \alpha_{k}\left\|-x^{k}\right\|\left\|x^{k}-y^{k}\right\| .
\end{aligned}
$$

Since $\lim _{k \rightarrow \infty} \alpha_{k}=0, \lim _{k \rightarrow \infty}\left\|x^{k}-x^{k+1}\right\|=0$, and $\lim _{k \rightarrow \infty}\left\|F\left(x^{k}\right)-F(z)\right\|=0$, we derive

$$
\lim _{k \rightarrow \infty}\left\|x^{k}-y^{k}\right\|=0 .
$$

(3) We show that $x^{k} \rightarrow z$.

By the property of the projection $P_{C}$, we have

$$
\begin{aligned}
& \left\|y^{k}-z\right\|^{2}=\| P_{C}\left[\left(1-\alpha_{k}\right)\left(x^{k}-\frac{\lambda_{k}}{1-\alpha_{k}} F\left(x^{k}\right)\right)\right] \\
& -P_{C}\left[\alpha_{k} z+\left(1-\alpha_{k}\right)\left(z-\frac{\lambda_{k}}{1-\alpha_{k}} F(z)\right)\right] \|^{2} \\
& \leq\left\langle-\alpha_{k} z+\left(1-\alpha_{k}\right)\right. \\
& \cdot\left[\left(x^{k}-\frac{\lambda_{k}}{1-\alpha_{k}} F\left(x^{k}\right)\right)-\left(z-\frac{\lambda_{k}}{1-\alpha_{k}} F\left(x^{k}\right)\right)\right], \\
& \left.y^{k}-z\right\rangle \leq \alpha_{k}\left\langle z, z-y^{k}\right\rangle+\left(1-\alpha_{k}\right) \\
& \cdot\left\|\left(x^{k}-\frac{\lambda_{k}}{1-\alpha_{k}} F\left(x^{k}\right)\right)-\left(z-\frac{\lambda_{k}}{1-\alpha_{k}} F(z)\right)\right\| \| y^{k} \\
& -z\left\|\leq \alpha_{k}\left\langle z, z-y^{k}\right\rangle+\left(1-\alpha_{k}\right)\right\| x^{k}-z\|\| y^{k}-z \| ;
\end{aligned}
$$

(36) then

$$
\begin{aligned}
\left\|y^{k}-z\right\|^{2} \leq & \alpha_{k}\left\langle z, z-y^{k}\right\rangle \\
& +\frac{1-\alpha_{k}}{2}\left[\left\|x^{k}-z\right\|^{2}+\left\|y^{k}-z\right\|^{2}\right] .
\end{aligned}
$$

Hence

$$
\left\|y^{k}-z\right\|^{2} \leq \frac{2 \alpha_{k}}{1-\alpha_{k}}\left\langle z, z-y^{k}\right\rangle+\frac{1-\alpha_{k}}{1+\alpha_{k}}\left\|x^{k}-z\right\|^{2} .
$$

Therefore,

$$
\begin{aligned}
\left\|x^{k+1}-z\right\|^{2} \leq & \left(1-\gamma_{k}\right)\left\|x^{k}-z\right\|^{2}+\gamma_{k}\left\|y^{k}-z\right\|^{2} \\
\leq & \left(1-\frac{2 \alpha_{k} \gamma_{k}}{1+\alpha_{k}}\right)\left\|x^{k}-z\right\|^{2} \\
& +\frac{2 \alpha_{k} \gamma^{k}}{1+\alpha_{k}} \frac{1+\alpha_{k}}{1-\alpha_{k}}\left\langle z, z-y^{k}\right\rangle .
\end{aligned}
$$


Since $0 \leq \lambda_{k} /\left(1-\alpha_{k}\right) \leq 2 / \rho\left(A^{T} A\right)$, we have

$$
0<\alpha_{k} \leq 1-\frac{\lambda_{k} \rho\left(A^{T} A\right)}{2} .
$$

Furthermore

$$
0<\frac{2 \alpha_{k} \gamma_{k}}{1-\alpha_{k}}<\frac{2 \gamma_{k}}{\rho\left(A^{T} A\right) \lambda_{k}}
$$

which implies

$$
\begin{aligned}
& \frac{2 \alpha_{k} \gamma^{k}}{1+\alpha_{k}} \frac{1+\alpha_{k}}{1-\alpha_{k}}\left\langle z, z-y^{k}\right\rangle \\
& \quad \leq \frac{2 \gamma_{k}}{\rho\left(A^{T} A\right) \lambda_{k}}\left\langle z, z-y^{k}\right\rangle .
\end{aligned}
$$

By condition (C4) and $\sum\left(\gamma_{k} / \lambda_{k}\right)<+\infty$ and $\left\langle z, z-y^{k}\right\rangle$ being bounded, we have $\sum_{k=1}^{\infty}\left(2 \gamma_{k} / \rho\left(A^{T} A\right) \lambda_{k}\right)\left\langle z, z-y^{k}\right\rangle<\infty$; hence, $\sum_{k=1}^{\infty}\left(2 \alpha_{k} \gamma^{k} /\left(1+\alpha_{k}\right)\right)\left(\left(1+\alpha_{k}\right) /\left(1-\alpha_{k}\right)\right)\left\langle z, z-y^{k}\right\rangle<\infty$. We apply Lemma 5 to inequality (44) to deduce that $x^{k} \rightarrow z$.

The following example of the SFP will show that our algorithm is feasible.

Example. Let $C=\left\{\left(x_{1}, x_{2}, x_{3}, x_{4}\right) \mid x_{1}+x_{2}-2 x_{4} \leq 0\right\}, Q=$ $\left\{\left(y_{1}, y_{2}\right) \mid y_{1}-y_{2} \leq 0\right\}$, and $A=\left[\begin{array}{llll}1 & 0 & 0 & 0 \\ 0 & 1 & 0 & 0\end{array}\right]$.

Select $x^{0}=(4,1,1,0)^{T}$ as a starting point for the algorithm. We take $\alpha_{k}=1 / k, \lambda_{k}=2 / \rho\left(A^{T} A\right) k^{2}, \gamma_{k}=1 / k^{4}$, and $\mu_{k}=2 / \rho\left(A^{T} A\right)\left(k^{4}+1\right)$. Then, for the method,

$$
\begin{aligned}
& x^{1}=(1.3103,0.8214,0,0.4275)^{T}, \\
& x^{2}=(0.4882,0.2135,0,0.1325)^{T}, \\
& x^{3}=(0.1213,0.0623,0,0.0513)^{T}, \\
& x^{4}=(0.0372,0.0129,0,0.0015)^{T}, \\
& x^{5}=(0.0012,0.0049,0,0.0020)^{T}, \\
& x^{6}=(0.0007,0.0015,0,0.0001)^{T}, \\
& x^{7}=(0.0000,0.0000,0,0.0000)^{T} .
\end{aligned}
$$

That is, the method obtains the approximate point $(0.0000,0.0000,0,0.0000)$ in seven steps.

\section{Conclusion}

In this paper, we presented a strongly convergent method for solving the split feasibility problem in Hilbert spaces inspired by the methods for solving the variational inequalities. Our results improve and develop previous split feasibility problem and related algorithms. Extension of this method for solving the multiple-set split feasibility problem is underway.

\section{Conflict of Interests}

The authors declare that there is no conflict of interests regarding the publication of this paper.

\section{Acknowledgments}

This work was supported by Natural Science Foundation of Shanghai (14ZR1429200), Innovation Program of Shanghai Municipal Education Commission (15ZZ074), and National Science Foundation of China (71572113).

\section{References}

[1] H. H. Bauschke and J. M. Borwein, "On projection algorithms for solving convex feasibility problems," SIAM Review, vol. 38, no. 3, pp. 367-426, 1996.

[2] Y. Censor and T. Elfving, "A multiprojection algorithm using Bregman projections in a product space," Numerical Algorithms, vol. 8, no. 2-4, pp. 221-239, 1994.

[3] C. Byrne, "A unified treatment of some iterative algorithms in signal processing and image reconstruction," Inverse Problems, vol. 20, no. 1, pp. 103-120, 2004.

[4] Y. Censor, "Parallel application of block-iterative methods in medical imaging and radiation therapy," Mathematical Programming, vol. 42, no. 2, pp. 307-325, 1988.

[5] C. Byrne, "Iterative oblique projection onto convex sets and the split feasibility problem," Inverse Problems, vol. 18, no. 2, pp. 441453, 2002.

[6] H. H. Bauschke, P. L. Combettes, and D. R. Luke, "A strongly convergent reflection method for finding the projection onto the intersection of two closed convex sets in a Hilbert space," Journal of Approximation Theory, vol. 141, no. 1, pp. 63-69, 2006.

[7] Y. Z. Dang and Y. Gao, "The strong convergence of a KM-CQlike algorithm for a split feasibility problem," Inverse Problems, vol. 27, no. 1, pp. 1-9, 2011.

[8] B. Qu and N. Xiu, "A note on the CQ algorithm for the split feasibility problem," Inverse Problems, vol. 21, no. 5, pp. 1655$1665,2005$.

[9] T. Suzuki, "Strong convergence theorems for infinite families of nonexpansive mappings in general Banach spaces," Fixed Point Theory and Applications, no. 1, pp. 103-123, 2005.

[10] F. Wang and H.-K. Xu, "Approximating curve and strong convergence of the CQ algorithm for the split feasibility problem," Journal of Inequalities and Applications, vol. 2010, Article ID 102085, 13 pages, 2010.

[11] H.-K. Xu, "A variable Krasnosel'skii-Mann algorithm and the multiple-set split feasibility problem," Inverse Problems, vol. 22, no. 6, pp. 2021-2034, 2006.

[12] Q. Yang, "The relaxed CQ algorithm solving the split feasibility problem," Inverse Problems, vol. 20, no. 4, pp. 1261-1266, 2004.

[13] J. Zhao and Q. Yang, "Several solution methods for the split feasibility problem," Inverse Problems, vol. 21, no. 5, pp. 17911799, 2005.

[14] E. Masad and S. Reich, "A note on the multiple-set split convex feasibility problem in Hilbert space," Journal of Nonlinear and Convex Analysis, vol. 8, no. 3, pp. 367-371, 2007.

[15] G. M. Korpelevich, "An extragradient method for finding saddle points and for other problems," Ekonomika i Matematicheskie Metody, vol. 12, no. 4, pp. 747-756, 1976. 
[16] N. Nadezhkina and W. Takahashi, "Weak convergence theorem by an extragradient method for nonexpansive mappings and monotone mappings," Journal of Optimization Theory and Applications, vol. 128, no. 1, pp. 191-201, 2006.

[17] L.-C. Ceng, Q. H. Ansari, and J.-C. Yao, "An extragradient method for solving split feasibility and fixed point problems," Computers \& Mathematics with Applications, vol. 64, no. 4, pp. 633-642, 2012.

[18] Y. Yao, M. Postolache, and Y.-C. Liou, "Variant extragradienttype method for monotone variational inequalities," Fixed Point Theory and Applications, vol. 2013, article 185, 2013.

[19] Y. Censor, A. Gibali, and S. Reich, "The subgradient extragradient method for solving variational inequalities in Hilbert space," Journal of Optimization Theory and Applications, vol. 148, no. 2, pp. 318-335, 2011.

[20] Y. Censor, A. Gibali, and S. Reich, "Strong convergence of subgradient extragradient methods for the variational inequality problem in Hilbert space," Optimization Methods and Software, vol. 26, no. 4-5, pp. 827-845, 2011.

[21] K. Goebel and S. Reich, Uniform Convexity, Hyperbolic Geometry, and Nonexpansive Mappings, Marcel Dekker, New York, NY, USA, 1984.

[22] W. Takahashi and M. Toyoda, "Weak convergence theorems for nonexpansive mappings and monotone mappings," Journal of Optimization Theory and Applications, vol. 118, no. 2, pp. 417428, 2003.

[23] H.-K. Xu, "Iterative algorithms for nonlinear operators," Journal of the London Mathematical Society, vol. 66, no. 1, pp. 240-256, 2002. 


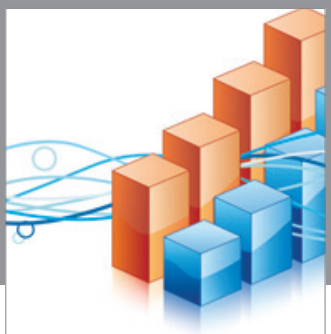

Advances in

Operations Research

vatem alat4

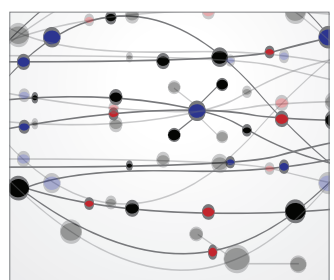

\section{The Scientific} World Journal
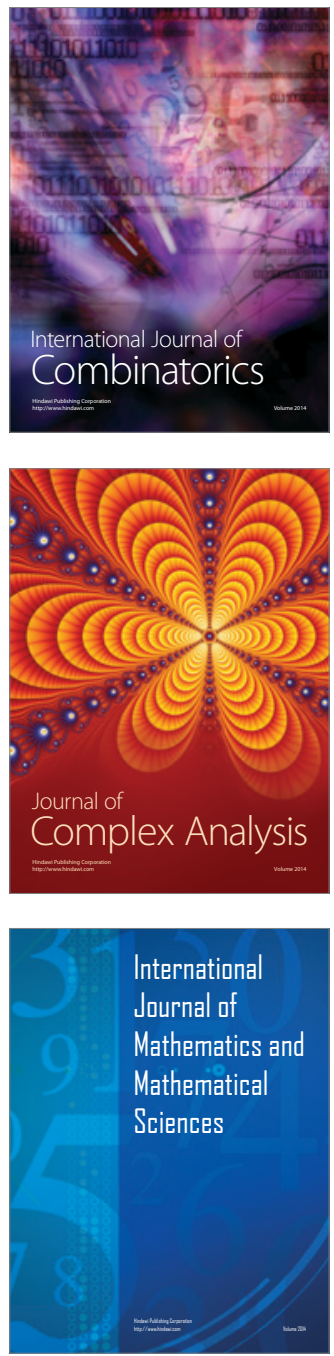
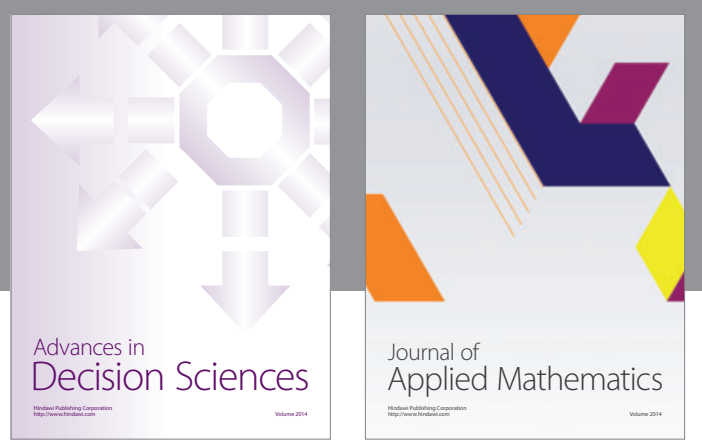

Algebra

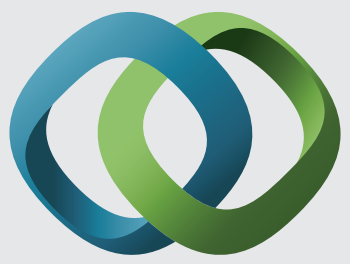

\section{Hindawi}

Submit your manuscripts at

http://www.hindawi.com
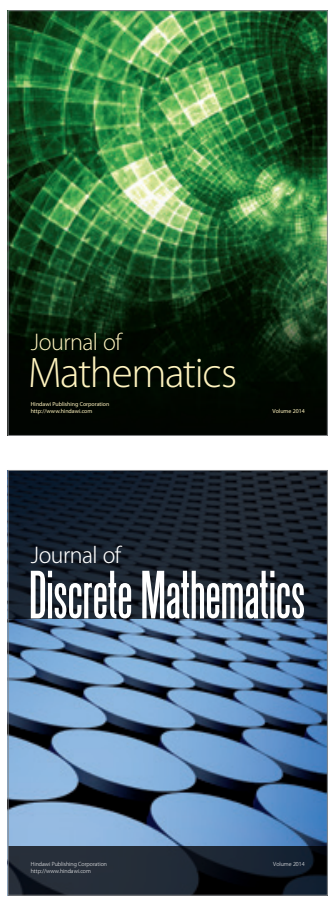

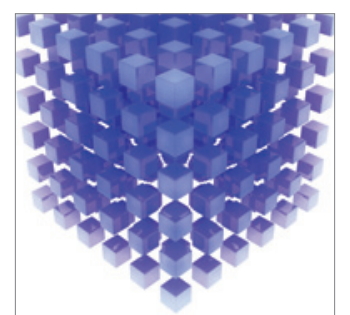

Mathematical Problems in Engineering
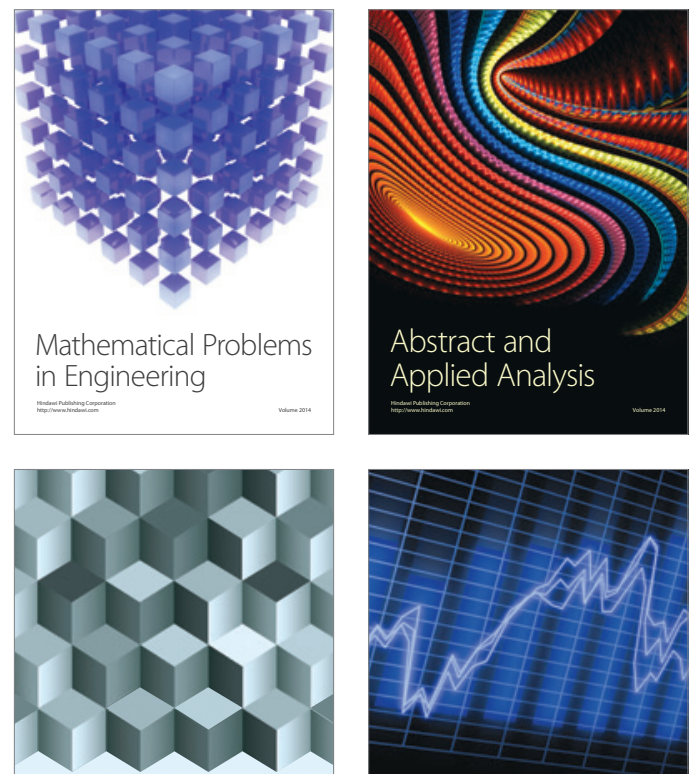

Journal of

Function Spaces

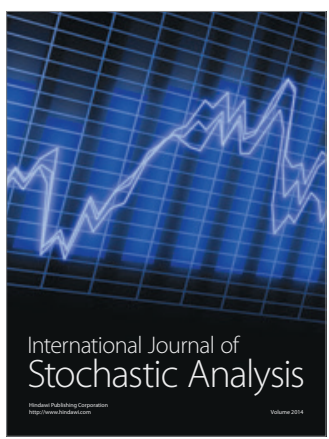

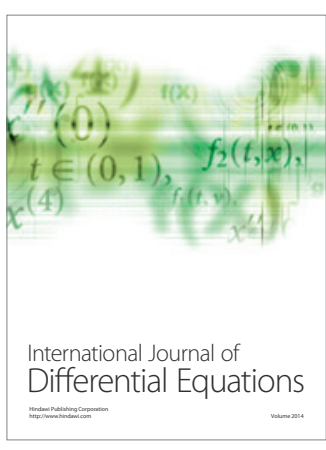
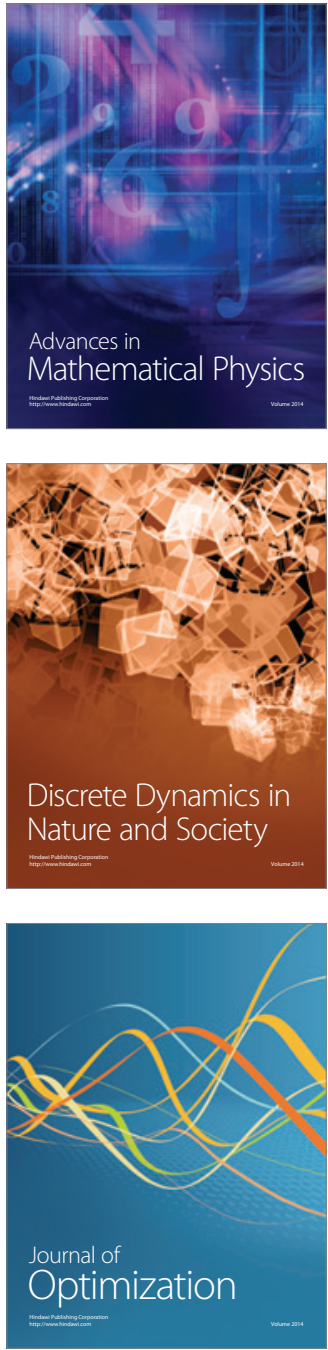